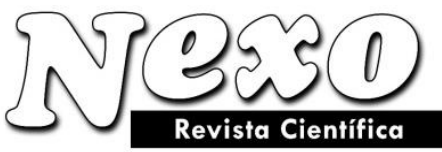

Universidad Nacional de Ingeniería COPYRIGHT @ (UNI). TODOS LOS DERECHOS RESERVADOS

http://revistas.uni.edu.ni/index.php/Nexo

https://doi.org/10.5377/nexo.v34i02.11547

Vol. 34, No. 02, pp. 616-624/Junio 2021

(c) (i) $(-)$

\title{
Selección de gasificador para la generación de energía eléctrica a pequeña escala empleando biomasa agricola
}

\section{Selection of gasifier for the generation of electrical energy at small scale using agricultural biomass}

\author{
Napoleón V. Blanco Orozco \\ Universidad Nacional de Ingeniería. Facultad de Electrotecnia y Computación, Departamento de Ingeniera \\ Eléctrica. Managua, Nicaragua \\ napoleon.blanco@fec.uni.edu.ni;blanconapoleon@yahoo.com
}

(recibido/received: 03-noviembre-2020; aceptado/accepted: 22-febrero-2021)

\begin{abstract}
RESUMEN
En este artículo se analizan los diferentes tipos de equipos de gasificación para la conversión de la biomasa de residuos agrícolas en energía eléctrica en pequeña escala. Se empleó la teoría fundamentada mediante un proceso metodológico que incluyó el análisis de citas, códigos, memos, familias de códigos y las relaciones entre estos elementos a través del examen de redes para estudiar los diferentes tipos de gasificadores y enlazar los conceptos que permitieran su selección para una aplicación específica. Además, se utilizó el método de comparación cualitativa mediante el empleo de indicadores y criterios técnicos para determinar que el gasificador de lecho móvil (down draft) resulta más apropiado para la generación de electricidad a partir de la gasificación de biomasa de residuos agrícolas en pequeña escala.
\end{abstract}

Palabras claves: Biomasa, Gasificación, Energía eléctrica.

\begin{abstract}
In this article, different types of gasification equipment for the conversion of biomass from agricultural residues into electrical energy on a small scale are analyze. The Grounded theory was used through a methodological process that included the analysis of citations, codes, memos, code families and the relationships between these elements through the examination of networks to study the different types of gasifiers and link the concepts that allowed their selection for a specific application. In addition, the qualitative comparison method was used with technical indicators and criteria to determine that the moving bed gasifier (down draft) is more appropriate for the generation of electricity from the gasification of biomass from agricultural residues on a small scale.
\end{abstract}

Keywords: Biomass, Gasification, Electrical energy.

\section{INTRODUCCIÓN}

La conversión energética de la biomasa para la generación de energía eléctrica puede realizarse mediante la quema directa para obtener vapor a disponer en turbinas o a través de la obtención de biocombustibles mediante la gasificación para obtener un gas que se puede disponer en motores de combustión interna 
acoplados a generadores. No obstante, el uso de un energético en forma de gas producto de la gasificación nos conduce a la pregunta de por qué utilizar el proceso de gasificación como etapa intermedia para luego quemar un gas de síntesis si por el contarios se puede obtener la energía de forma directa y la respuesta la refiere Ruiz (2014) planteando que "los rendimientos en funcionamientos estables del proceso de gasificación son superiores a la combustión" por lo que, en este escrito nos ocupamos de la selección de la tecnología de gasificación para la conversión energética de la biomasa para pequeñas capacidades en sistemas de generación distribuida.

De manera que, la gasificación de la biomasa es un proceso termoquímico que tiene como fin la transferencia de energía contenida en el combustible sólido a un portador energético gaseoso; de forma tal que, transferir a un portador energético en forma de gas la energía química contenida en la biomasa es el propósito de la gasificación (Travieso, 2007) y la gasificación es un proceso químico cuyo propósito es la transferencia máxima de energía química del combustible al gas producto (Paredes y Baca, 2005). Por lo tanto, la biomasa puede ser transformada en combustibles líquidos, sólidos o gaseosos para usarse en máquinas térmicas con una eficiencia alrededor del veinte por ciento (Quintero et al., 2018).

Así mismo, la gasificación es definida como un proceso que convierte combustibles fósiles en gases combustible y al gas resultante se le llama syngas o sintergas (Ruiz, 2014; Estrada y Zapata, 2004). Y el gas de síntesis producto de la gasificación de biomasa es útil si su poder calorífico tiene un contenido energético especifico que se referencia por encima de los cuatro mega joule por Newton metro (Cabrera et al., 2013). Por otra parte, la gasificación puede realizarse con un equipo llamado gasificador y en la literatura se determinan los tipos siguientes de gasificadores: lecho móvil, lecho fijo (updraft-contracorriente o downdraft corrientes paralelas) y lecho fluidizado. Así que, para seleccionar el tipo de gasificado óptimo para la generación de energía eléctrica en pequeña escala pueden considerar factores técnicos y ambientales como la limpieza del gas, tamaño del residuo energético empleado y su humedad. El propósito de este artículo es la revisión de las tecnologías disponibles de gasificación de la biomasa residual agrícola para la selección de un gasificador que pueda utilizarse para la generación de electricidad en pequeña escala.

\section{METODOLOGÍA}

El proceso metodológico utilizado en este trabajo fue del tipo cualitativo, se emplearon preceptos de la teoría fundamentada sustentada en referentes teóricos como lo fue la revisión del estado del arte de la gasificación de residuos agrícolas para la producción de energía eléctrica utilizando la herramienta informática Atlas ti versión número siete para el desarrollo del análisis documental. El proceso de sustentación incluyó el análisis de citas, códigos, memos, familias de códigos, y las relaciones entre estos elementos a través del examen de redes hasta llegar a la saturación teórica con el propósito de realizar una puesta en común de enfoques de la literatura consultada para determinar la tecnología de gasificación que se ajuste a ciertos criterios de selección para aplicaciones de pequeña potencia.

Así que, se desarrolló un análisis de citas y códigos relacionando los elementos coincidentes en el procesamiento de la información documental y de esta forma se encontraron puntos en común referentes y a partir de allí se procedió con un análisis de red con el propósito de enlazar los conceptos que conducen a la selección del tipo de gasificador que se ajuste a la aplicación de generación de energía eléctrica de pequeña potencia con residuos biomásicos agrícolas y que cumple en mayor proporción con factores técnicos y ambiental seleccionados.

Adicionalmente, para la revisión y selección de la tecnología para la conversión energética de la biomasa se empleó el método de comparación contraste de forma tabular; para lo cual, se seleccionaron indicadores técnicos y ambientales para comparar los tipos de gasificadores y recomendar aquel que más se ajusta para la aplicación específica de generación de energía eléctrica a pequeña escala. De forma que, los indicadores técnicos fueron seleccionados del marco teórico con la ayudad del programa Atlas ti al ser los indicadores 
más utilizados, utilizados con mayor frecuencia, en los artículos científicos estudiados; de forma que, se obtuvo una comparación cualitativa de los índices técnicos para la evaluación de los tipos de gasificadores. Finalmente, se logró la selección del equipo de gasificador que más se ajusta a la aplicación específica de pequeña potencia para la generación de energía eléctrica con biomasa residual agrícola.

\section{RESULTADOS}

\subsection{Tecnología para la conversión energética de la biomasa}

Según el análisis de citas, códigos y su relación en la unidad hermenéutica de los gasificadores analizada con Atlas ti para la gasificación de la biomasa se dispone en la actualidad de los siguientes tipos de gasificadores que se observan en la figura 1 y que se detallan a continuación:

El gasificador de lecho fijo que es considerado apropiado para para sistemas con eficiencias globales cercanas al veinte por ciento lo que significa una conversión de 1,2 kilogramos para generar un kilo watts hora de energía eléctrica (Quintero et al., 2018) y este tipo de máquina es usada en la gasificación de biomasa sobre todo para bajas potencias entre 10 a 100 Kilo watts de potencia eléctrica (Yiepes y Farid, 2012). Así mismo, este tipo de gasificador produce gas con poco contenido de alquitrán, el cual se puede limpiar empleando tanques de filtración (Asprilla, 2018). Además, este gasificador es recomendado cuando la biomasa es reducida a pequeñas partículas y de baja humedad y son utilizados principalmente en plantas de generación de energía de pequeñas potencias (Ruiz, 2014).

Desde el punto de vista de los costos de inversión, Forero, Guerrero y Sierra (2012) manifiestan que los reactores de lecho fijo son equipos utilizados en países en vías de desarrollo y en los que no pueden realizar grandes inversiones ni asumir costos elevados para la operación y el control de los equipos. En este mismo sentido, Balderrama et al (2011) expresan que, si se desea que la operación de gasificadores con capacidades de $400 \mathrm{~kW}$ sea rentable, para su operación se deben facilitar subsidios y otros beneficios adicionales a los ingresos por venta de energía eléctrica tales como ingresos por bonos de carbono.

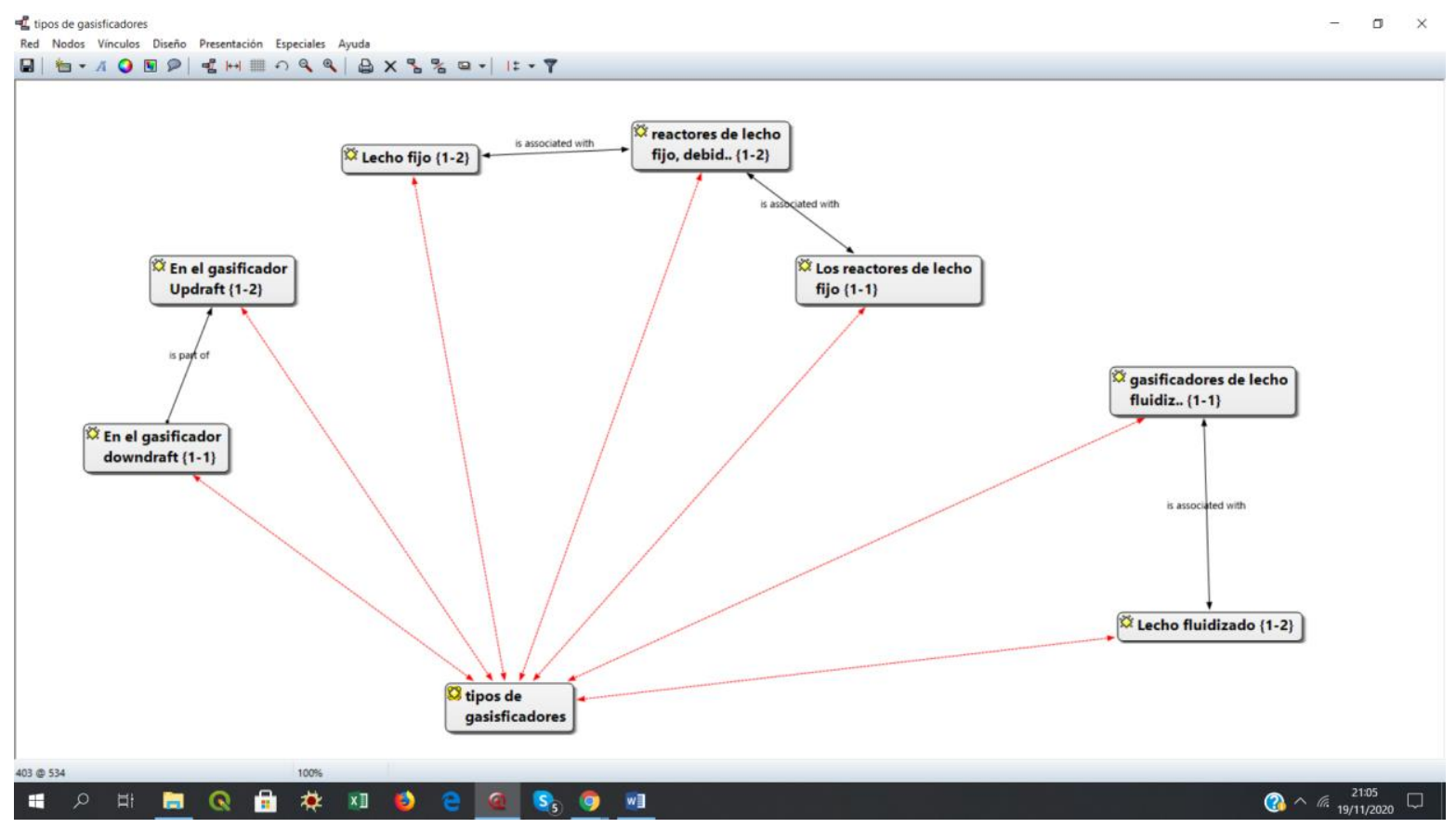

Figura 1. Tipos de gasificadores indicados en el análisis de códigos usando Atlas ti. 
El gasificador de lecho fluidizado que es empleado para la generación de electricidad a gran escala, generalmente para capacidades superiores a los $100 \mathrm{~kW}$ y produciendo menor cantidad de contaminantes como alquitranes y cenizas (Yiepes y Farid, 2012). Así, este gasificador produce mayores velocidades de reacción y eficiencias de conversión y control adecuado de temperatura; generando al final niveles moderados de alquitrán (García, 2016)

. En el mismo sentido, estos equipos desarrollan la combustión con masa en suspensión de partículas de combustible y cenizas que son fluidizado por una corriente ascendente de aire; pero tienen la capacidad de gasificar diferentes tipos de biomasa (Ruiz, 2014).

El gasificador de lecho móvil downdraft que se recomienda para aplicaciones de generación de electricidad de pequeñas capacidades menores a un mega watts de potencia, debido a su capacidad de producción de gas suministrado a los motores de combustión interna (Lesme-Jaén, 2016; Suárez et al., 2017). De la misma forma, Vega y Herrera (2017) citando a Held (2012) plantean que los gasificadores de corriente descendente son adecuados para capacidades entre uno y uno y medio mega watts de potencia. En este sentido, Castellanos et al. (2011) consideran que este tipo de gasificador es "más aceptable para aplicaciones en motores de combustión interna y turbinas de gas de combustión interna". Por otra parte, Estrada y Zapata (2004) señalan como desventaja de este equipo la producción de ceniza y las condiciones de humedad en el reactor.

Los gasificadores de lecho móvil corriente ascendente (updraft) que se consideran adecuados para sistemas con potencia entre cuatro y seis mega watt (Vega y Herrera, 2017). Así mismo, este tipo de gasificadores producen alto contenido de alquitrán y presentan imposibilidad de generar gas de síntesis en forma permanente, por el contrario, su capacidad de producción es fluctuante haciendo difícil su aplicación en motores de combustión interna (Castellanos et al.,2011). Con respecto a la producción de residuos este gasificador es recomendado para biomasa que genere gran cantidad de cenizas (Ruiz, 2014). Por otra lado, Estrada y Zapata (2004) describen que este gasificador es de alta eficiencia en su proceso de debido a su diseño; no obstante, presenta producción de alquitranes y problemas con la capacidad de producción continua del gas de salida.

De manera general, se recomienda la gasificación de la biomasa en plantas con capacidad entre 500 kilowatts y dos mega watts y para capacidades superiores se considere la combustión directa (Ruiz, 2014). Adicionalmente, el gas producido en el proceso de gasificación debe tener algunas propiedades como un contenido de alquitranes y polvo menor que 10 miligramos por Newton metro cubico; de no ser así, se tendría que recurrir a sistemas de limpieza (Travieso, 2007).

\subsection{Criterios para la selección de gasificadores para la generación de energía en pequeña escala}

Con el propósito de seleccionar entre los tipos de gasificadores referenciados por la literatura revisada el que más se ajusta a la aplicación de pequeñas plantas de generación de energía eléctrica en sistemas de generación distribuida se ha procedido a seleccionar criterios técnicos para caracterizar y comparar los diferentes tipos lo que se resume en la tabla número uno.

Tabla 1. Indicadores y criterios técnicos para la selección de las tecnologías para la conversión energética de la biomasa para aplicaciones de pequeña potencia.

\begin{tabular}{ccccc}
\hline Criterios & $\begin{array}{c}\text { Lecho móvil updraft- } \\
\text { contracorriente }\end{array}$ & $\begin{array}{c}\text { Lecho móvil } \\
\text { down draft }\end{array}$ & Lecho fijo & Lecho fluidizado \\
\hline Madurez & Reactor sencillo & $\begin{array}{c}\text { Tecnología } \\
\text { simple y } \\
\text { probada } \\
\text { tecnológica }\end{array}$ & $\begin{array}{c}\text { Tecnología } \\
\text { sencilla }\end{array}$ & $\begin{array}{c}\text { Tecnología reciente, } \\
\text { compleja }\end{array}$ \\
$\begin{array}{c}\text { Humedad máxima } \\
\text { de la biomasa }\end{array}$ & $60 \%$ & Baja humedad & $\begin{array}{c}\text { Admite una alta } \\
\text { variación en la } \\
\text { humedad }\end{array}$ \\
$\begin{array}{c}\text { Contaminación } \\
\text { ambiental, }\end{array}$ & $\begin{array}{c}\text { Alta cantidad de } \\
\text { cenizas y alquitrán }\end{array}$ & $\begin{array}{c}\text { Bajos } \\
\text { contenidos de } \\
\text { alquitranes y } \\
\text { cenizas }\end{array}$ & $\begin{array}{c}\text { Poco contenido de } \\
\text { alquitrán }\end{array}$ & $\begin{array}{c}\text { Niveles moderados } \\
\text { de alquitrán generado }\end{array}$ \\
generado & & $\begin{array}{c} \\
\end{array}$ & &
\end{tabular}




\begin{tabular}{|c|c|c|c|c|}
\hline Criterios & $\begin{array}{l}\text { Lecho móvil updraft- } \\
\text { contracorriente }\end{array}$ & $\begin{array}{l}\text { Lecho móvil } \\
\text { down draft }\end{array}$ & Lecho fijo & Lecho fluidizado \\
\hline $\begin{array}{l}\text { Eficiencia de la } \\
\text { combustión o } \\
\text { rendimiento }\end{array}$ & Alta & Alta & Baja & Alta \\
\hline $\begin{array}{l}\text { Costo de } \\
\text { inversión en } \\
\text { equipos }\end{array}$ & Altos & Bajos & Bajo & Altos \\
\hline $\begin{array}{l}\text { Potencias } \\
\text { admisibles }\end{array}$ & Hasta $10 \mathrm{MW}$ & Hasta $1 \mathrm{MW}$ & de 10 a $100 \mathrm{KW}$ & 2 a $50 \mathrm{MW}$ \\
\hline Uso & $\begin{array}{c}\text { Indicados para } \\
\text { aplicaciones del gas } \\
\text { en generación de } \\
\text { calor. } \\
\text { Capacidad marginal } \\
\text { que tiene en su carga, } \\
\text { imposibilidad de } \\
\text { generar de un gas de } \\
\text { síntesis permanente si } \\
\text { no fluctuante } \\
\text { aumentando las } \\
\text { dificultades para la } \\
\text { utilización en } \\
\text { motores }\end{array}$ & $\begin{array}{c}\text { Utilizados para } \\
\text { el uso en } \\
\text { motores de } \\
\text { combustión } \\
\text { interna a } \\
\text { pequeña escala } \\
\\
\text { Para la } \\
\text { generación de } \\
\text { electricidad a } \\
\text { pequeña (< } \\
1 \mathrm{MW}) .\end{array}$ & $\begin{array}{l}\text { Aplicaciones en } \\
\text { regiones apartadas } \\
\text { de } 10 \text { a } 100 \mathrm{KW} .\end{array}$ & $\begin{array}{c}\text { Generación a gran } \\
\text { escala, generalmente } \\
\text { para } \\
\text { capacidades } \\
\text { superiores a los } \\
100 \mathrm{~kW}\end{array}$ \\
\hline
\end{tabular}

De forma que, al realizar un análisis de red de relación de códigos y la familia de la literatura consultada relacionada a los tipos de gasificadores se obtiene que efectivamente la generación de electricidad puede obtenerse a través de la gasificación que producirá un gas de síntesis y que el gasificador puede ser de diferentes tipos a como se detalla en la figura número dos.

Además, considerando los criterios analizados en la tabla número uno se tiene que el gasificador de lecho móvil down draft es más apropiado para el uso en la gasificación de biomasa de residuos agrícolas en pequeña escala debido a que es una tecnología sencilla pero probada, con bajo niveles de emisión de contaminantes, alta eficiencia en la conversión energética, costos de inversión bajo y que pueden ser empleados en pequeños motores de combustión interna para acoplarse a generadores de energía eléctrica. 


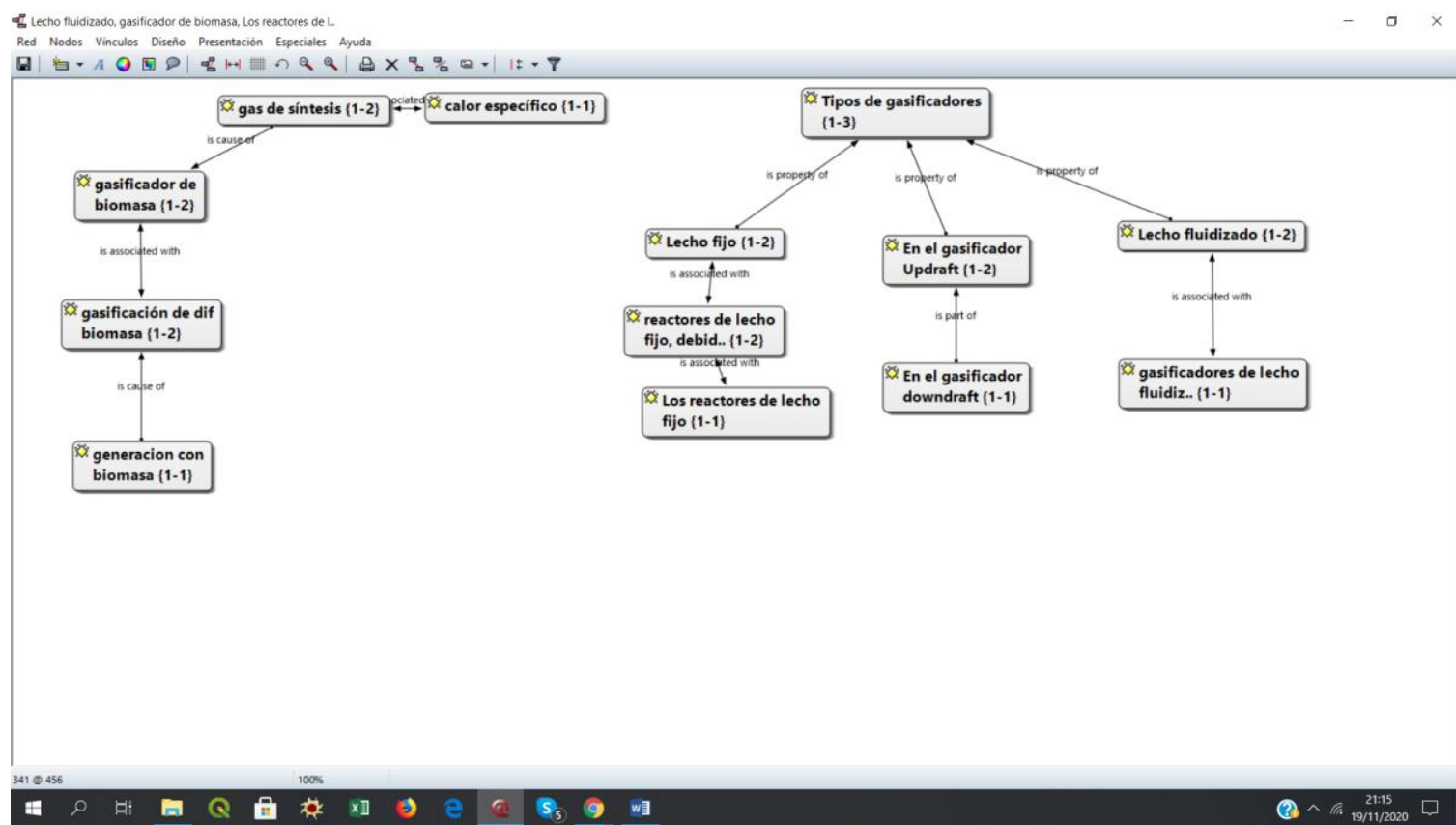

Figura 2. Análisis de red de la gasificación de la biomasa usando Atlas ti.

\subsection{Tipo de motor a utilizar en el generador eléctrico}

Para la generación de energía eléctrica en conjunto con un gasificador existe la posibilidad de emplear motores de combustible mixto gas-diésel o motores de encendido por chispa.

Para aplicaciones de generadores de pequeña potencia se puede utilizar de forma acoplada el motor de combustión interna con chispa que puede funcionar con gas pobre. No obstante, la potencia de los motores de combustión interna presentará una reducción de su potencia hasta en un cincuenta por ciento; por lo que, se deben de implementar modificaciones en la relación de compresión del motor y su velocidad (Ruiz, 2014). En el caso de los motores de combustión interna que operan con combustible diésel presentan la dificultad de que junto al gas pobre se debe utilizar una porción de diésel dado que, el gas pobre del gasificador, no es capaz de inflamarse por la compresión (Ídem).

\subsection{Proceso adicional para aumentar la densidad de la biomasa}

Con el fin de obtener un combustible energético biomásico para su aplicación en la gasificación con propiedades físicas homogéneas y aumentar la densidad calorífica, se utilizan procesos como el astillado, briquetado y peletizado. La densificación de la biomasa a través del briquetado y peletizado permite obtener un combustible con propiedades homogéneas y mayor poder calorífico (Torres et al., 2018; Ruiz, 2014). Así, el proceso de astillado se logra a través de la trituración de la madera para obtener pequeños (Martínez, 2009). Por otra parte, el briquetado es un proceso que consiste en la compresión de la biomasa para conferirle una densificación mayor y el peletizado es también la compresión de biomasa, pero con rodillos contra una superficie con agujeros y así obtener pequeños trozos o pellets (Ruiz, 2014).

\section{CONCLUSIONES}

Este articulo tuvo el fin de desarrollar una revisión de las tecnologías disponibles de gasificación de la biomasa residual agrícola para la selección de un gasificador que pueda utilizarse para la generación de electricidad en pequeña escala. De forma que, se concluye que la generación de energía eléctrica mediante el empleo de la biomasa se puede realizar con su quema directa o a través de la gasificación de este combustible. Además, con el fin de convertir a la biomasa en un energético con propiedades físicas 
homogéneas y aumentar su densidad calorífica se recomienda utilizar procesos como el astillado, briquetado y peletizado. Por otro lado, el proceso de gasificación de la biomasa puede realizarse con gasificadores de lecho móvil, de lecho fijo y lecho fluidizado.

La teoría fundamentada es útil para realizar un análisis de la literatura técnica de un área específica y en el caso de este artículo se utilizó para obtener la puesta en común de criterios de selección de la tecnología de gasificación que más se ajustan a la generación de energía eléctrica de pequeña potencia utilizando biomasa residual proveniente de la producción agrícola.

Así que, se empleó la teoría fundamentada con el propósito de encontrar a través de un estudio de citas, códigos y análisis de red el tipo de gasificador que se ajusta a la aplicación de generación de energía eléctrica con residuos biomásicos agrícolas. Además, en combinación con el uso de la teoría fundamentada se empleó el método de comparación contraste cualitativo para seleccionar indicadores técnicos y de esta forma se logró la comparación de diferentes tipos de gasificadores. Finalmente, con los criterios analizados se determinó que el gasificador de lecho móvil down draft resulta más apropiado para la gasificación de biomasa de residuos agrícolas en pequeña escala.

\section{REFERENCIAS}

Rolland, L. (2005). Certified solving of the forward kinematics problem with an exact algebraic method for the general parallel manipulator. Advanced Robotics, 19 (9), 995-1025.

Ahumada, L; Verdeza, A; Bula, A; Lombana, J. Optimización de las Condiciones de Operación de la Microgasificación de Biomasa para Producción de Gas de Síntesis. 2016. Información Tecnológica, Vol. 27 Issue 3, 179-188. 10p. DOI: 10.4067/S0718-07642016000300017. Obtenido desde: http://web.a.ebscohost.com/ehost/pdfviewer/pdfviewer?vid=10\&sid=210d8971-a41c-4fb4-8e7247e 5d7a92ce0\%40sdc-v-sessmgr02

Asprilla D. (2018). Estudios de Sistemas Híbridos de Energía Renovable (solar - gasificación de biomasa) como alternativa para satisfacer necesidades energéticas en Zonas no Interconectadas del Departamento del Chocó. Tesis presentada como requisito parcial para optar al título de: Magister en Ingeniería Mecánica. Universidad Nacional de Colombia Facultad de Minas Medellín, Colombia.

Balderrama-Castañeda, S; Luján-Álvarez, C; Lewis, D; Ortega-Gutiérrez, J; Jong, B.; Nájera-Ruiz, T. Madera y Bosques. (2011). Factibilidad de generación de electricidad mediante gasificación de residuos de aserradero en el norte de México. Madera y Bosques 17(2), 67-84. Obtenido desde:http://web.a.ebscohost.com/ehost/pdfviewer/pdfviewer? vid=8\&sid=210d8971-a41c-4fb4-8e7247e $5 \mathrm{~d} 7 \mathrm{a} 92 \mathrm{ce} 0 \% 40$ sdc-v-sessmgr02

Cabrera, G; Madriñan, S y Muñoz D. (2013). Evaluación de índices de conversión energética en la producción de gas combustible a partir de biomasa. Biotecnología en el Sector Agropecuario y Agroindustrial, Edición Especial (2), $118 \quad-\quad 125 . \quad$ Obtenido desde: http://web.a.ebscohost.com/ehost/pdfviewer/pdfviewer?vid=5\&sid=210d8971-a41c-4fb4-8e7247e5d7a92ce0\%40sdc-v-sessmgr02

Castellanos, J; Sierra, F y Guerrero, C. (2011). Modelamiento del proceso de gasificación de biomasa para aprovechamiento energético: una revisión al estado del arte. Ingenium. Vol. 12 Issue 23, 5-18. 14p. Obtenido desde: http://web.a.ebscohost.com/ehost/pdfviewer/pdfviewer?vid=24\&sid=210d8971-a41c-4fb4-8e7247e5d7a92ce0\%40sdc-v-sessmgr02 
Gómez, R.; Camargo, D; Soto, C.; Bula, A. (2019). Evaluación Sinérgica de la Gasificación de Biomasa Residual en Mezclas de Maíz y Algodón. Información Tecnológica, 30 (6), 11-20. 10p. DOI: 10.4067/S0718-07642019000600011.

Obtenido

desde:http://web.a.ebscohost.com/ehost/pdfviewer/pdfviewer? vid=9\&sid=210d8971-a41c-4fb4-8e72$47 \mathrm{e} 5 \mathrm{~d} 7 \mathrm{a} 92 \mathrm{ce} 0 \% 40$ sdc-v-sessmgr02

Durán-García, M.2016. Gasificación del aserrín de pino (pinus arizonica) en un reactor de lecho fluidizado: análisis de las variables del proceso. Maderas: Ciencia y Tecnología, 18 (4), p633-650. 18p. Obtenido desde: http://web.a.ebscohost.com/ehost/pdfviewer/pdfviewer?vid=17\&sid=210d8971-a41 c-4fb4-8e72-

47e5d7a92ce0\%40sdc-v-sessmgr02

Estrada, C y Zapata A. (2004). Gasificación de biomasa para producción de combustibles de bajo poder calorífico y su utilización en generación de potencia y calor. Scientia et Technica Año X, 25.

Guerrero-Ordóñez, J, Pantoja-Bucheli, A, Castillo-Muñoz, J. (2016). Metodología para el aprovechamiento del potencial energético con biomasa forestal en el departamento de Nariño: caso de estudio. Ingeniería Solidaria, 12 (20). DOI: http://dx.doi.org/10.16925/in.v19i20.1415

Lesme-Jaén, R; Garcia-Faure, L; Oliva-Ruiz, L; Pajarín-Rodríguez, J; Revilla-Suare, D. (2016). Gasificación de biomasa para la generación de electricidad con motores de combustión interna. Tecnología Química, $36 \quad$ (2), 161-172. 12p. Obtenido desde: http://web.a.ebscohost.com/ehost/pdfviewer/pdfviewer?vid=4\&sid=210d8971-a41c-4fb4-8e7247e5d7a92ce0\%40sdc-v-sessmgr02

Martínez, S. (2009). Evaluación de la biomasa como recurso energético renovable en Cataluña. Tesis doctoral. Universitat de Girona. España

Martillo, J; Lesme, R; Martínez, A; Oliva, O y Orozco, L. Estudios paramétricos de la gasificación de la tusa de maíz en gasificadores downdraft. Tecnología Química, 39 (2), 455-470. 16p. Obtenido desde: http://web.a.ebscohost.com/ehost/pdfviewer/pdfviewer?vid=29\&sid=210d8971-a41c-4fb4-8e72-

47e $5 \mathrm{~d} 7 \mathrm{a} 92 \mathrm{ce} 0 \% 40$ sdc-v-sessmgr02

Paredes, R y Baca, M.2005.Generación de energía con biogás de residuos agrícolas en plantas agroindustriales la libertad - Perú. Third LACCEI International and Caribbean for Engineering and Technology (LACCET' 2005) “Advances in Engineering and Technology: A Global Perspective”, p8 - 10. Cartagena de la India, Colombia.

Quintero Coronel, Y; Rodas, L y Corredor, L.2018. "Desarrollo de un modelo de gasificación en equilibrio químico para evaluar el potencial energético del cuesco en plantas extractoras de aceite de palma en Colombia,” INGE CUC, 14 (2), 62-70. DOI: http://doi.org/10.17981/ingecuc.14.2.2018.06

Ruiz J. (/2014). Análisis de la problemática e investigación de aspectos avanzados de la generación eléctrica con biomasa. Tesis Doctoral. Escuela Técnica Superior de Ingeniería Industrial. Universidad de la Rioja.

Suárez, L; Pérez, J; Barrera, R.2017. Gasificación de madera para la obtención de un syngas útil en la producción de biocombustibles y/o productos químicos. Revista ION. 30 (1), 57-71. 15p. DOI: 10.18273/revion. v30n1-2017005. Obtenido desde: http://web.a.ebscohost.com/ehost/pdfviewer/pdfviewer?vid=21\&sid=210d8971-a41c-4fb4-8e7247e $5 \mathrm{~d} 7 \mathrm{a} 92 \mathrm{ce} 0 \% 40$ sdc-v-sessmgr02 
Torres, C; Chávez M; Urbina L y Moya R. 2018. Evaluación de la incidencia de pellets y astillas de madera en el desempeño de un gasificador tipo "downdraft". Revista Forestal Mesoamericana Kurú ,15. 25-36. DOI: $10.18845 /$ rfmk.v15i1.3847

Travieso, D. (2007). Perspectives of Electricity Generation in Cuba from Biomass Gasification. Revista de

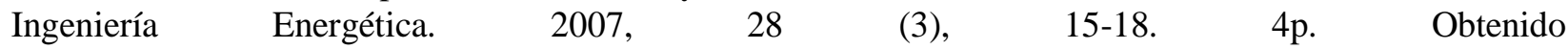
desde:http://web.a.ebscohost.com/ehost/pdfviewer/pdfviewer?vid=11\&sid=210d8971-a41c-4fb4-8e7247e5d7a92ce0\%40sdc-v-sessmgr02

Vega, L., y Herrera, A. (2018). Evaluación del potencial energético de la madera residual urbana mediante gasificación. Informador Técnico, 82(1), 26-40. DOI: http://doi.org/10.23850/22565035.888

Yepes D y Farid, J .2012. Gasificación de la biomasa residual en el sector floricultor, caso: oriente

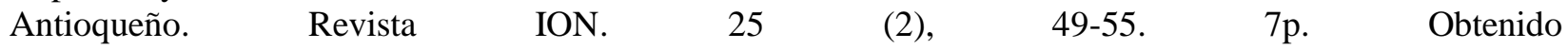
desde:http://web.a.ebscohost.com/ehost/pdfviewer/pdfviewer?vid=26\&sid=210d8971-a41c-4fb4-8e7247e $5 \mathrm{~d} 7 \mathrm{a} 92 \mathrm{ce} 0 \% 40$ sdc-v-sessmgr02

\section{SEMBLANZA DE LOS AUTORES}

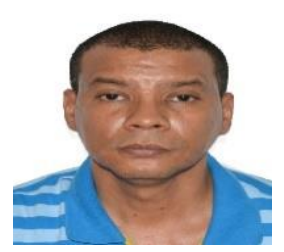

Napoleon V. Blanco Orozco: Doctor en Ciencias del Instituto Tecnológico de Costa Rica (ITCR) en 2015. Máster en Gestión de Proyectos en la UNI, 2009. Ingeniero eléctrico graduado en 1998 en la UNI. Es profesor de la Universidad Nacional de Ingenieria en el área de metodología de la investigación, máquinas eléctricas y la gestión de proyectos, Jefe del departamento y coordinador del grupo de investigación de ingeniería eléctrica en la UNI en la Facultad de Electrotecnia y Computación. 